\title{
554 - Evaluation of CPR Decisions on an Older Adult Psychiatric Ward, A Quality Improvement Project
}

Bennett L, McKinlay M, Prasanna A

Word Count: 350

\section{Background}

The National Confidential Enquiry into Patient Outcomes and Death (NCEPOD) stated that CPR status must be considered and recorded for all acute hospital admissions. Compliance with this recommendation and with Do Not Attempt Cardiopulmonary Resuscitation (DNACPR) form documentation in an inpatient psychiatric hospital was assessed. Multidisciplinary team (MDT) opinions surrounding DNACPR were also explored with an aim to educate staff, improving frequency and quality of future discussions.

\section{Research Objective}

Ensure patient suitability for CPR is discussed at admission, that discussions are documented and forms completed in line with Trust policy and national guidelines.

\section{Method}

Resuscitation Council UK guidelines were used as a standard, namely recommendations for clear and full documentation of CPR decisions triggered by a new admission to hospital. A retrospective study of admissions to the older adult psychiatric ward over a four-month period was carried out, identifying 25 patients fulfilling the inclusion criteria. Demographics and CPR consideration at the initial consultant ward round were documented. Completed DNACPR forms were audited for compliance with Trust guidance. Following data collection, 14 staff interviews using standardised questions were completed to gauge understanding of DNACPR. Answers were analysed and education was identified as key. Bite-sized teaching for MDT staff on DNACPR was carried out and response to the intervention assessed using these same standardised questions.

\section{Preliminary Results of the Ongoing Study}

1 patient out of 25 had a CPR discussion documented from their initial consultant review. $12 \%$ had documentation of DNACPR consideration throughout the entirety of admission. The 1 DNACPR form subsequently completed had $91 \%$ compliance with Trust policy. Qualitative results from staff interviews were insightful with 50\% knowing where DNACPR forms werekept, $29 \%$ feeling confident discussing DNACPR and 93\% feeling able to contribute to team decisions. Following a bite-sized education session these figures increased to $100 \%$ having awareness and confidence discussing CPR suitability.

\section{Conclusion}

DNACPR considerations are infrequent and staff interviews suggest this may be due to lack of confidence and knowledge surrounding CPR. Bite-sized education may play a significant role in informing the MDT and ensuring vital DNACPR considerations are not forgotten about in the psychiatric setting.

\section{5 - Complex Visual and Haptic Hallucinations in an Elderly Patient... A diagnostic challenge Joana Regala ${ }^{1}$, Camila Nóbrega $^{2}$, João Reis $^{3}$ \\ 1-Centro Hospitalar Psiquiátrico de Lisboa (CHPL), Lisbon, Portugal. \\ 2-Department of Neurology and Neuropsychiatry, Centro Hospitalar Psiquiátrico de Lisboa (CHPL), Lisbon, Portugal. \\ 3-Department of Geriatric Psychiatry, Centro Hospitalar Psiquiátrico de Lisboa (CHPL), Lisbon, Portugal.}

Background:

Hallucinations are common in elderly. Understanding the diverse aetiologies and behavioural reactions to hallucinations is vital to provide the best level of care. 